\title{
ANOMALOUS DIFFUSION OF SOLAR MAGNETIC ELEMENTS
}

\author{
A. C. Cadavid ${ }^{1}$ and J. K. Lawrence \\ Department of Physics and Astronomy, California State University, Northridge, CA 91330 \\ AND \\ A. A. RuZmaikin \\ Jet Propulsion Laboratory, California Institute of Technology, 4800 Oak Grove Drive, Pasadena, CA 91109 \\ Received 1998 October 9; accepted 1999 March 30
}

\begin{abstract}
The diffusion properties of photospheric bright points associated with magnetic elements (magnetic bright points) in the granulation network are analyzed. We find that the transport is subdiffusive for times less than 20 minutes but normal for times larger than 25 minutes. The subdiffusive transport is caused by the walkers being trapped at stagnation points in the intercellular pattern. We find that the distribution of waiting times at the trap sites obeys a truncated Lévy type (power-law) distribution. The fractal dimension of the pattern of sites available to the random walk is less than 2 for the subdiffusive range and tends to 2 in the normal diffusion range. We show how the continuous time random walk formalism can give an analytical explanation of the observations. We simulate this random walk by using a version of a phenomenological model of renewing cells introduced originally for supergranules by Simon, Title, \& Weiss. We find that the traps that cause the subdiffusive transport arise when the renewed convection cell pattern is neither fixed nor totally uncorrelated from the old pattern, as required in Leighton's model, but in some intermediate state between these extremes.
\end{abstract}

Subject headings: diffusion - Sun: granulation — Sun: magnetic fields

\section{INTRODUCTION}

A key to understanding solar surface phenomena is determining how photospheric convective motions transport magnetic flux elements. A central process of this transport is diffusion, taken as the cumulative effect of random walks. The properties of the solar diffusion provide both insight into the evolution of surface magnetic fields and information about the properties of the underlying velocity field.

The random character of solar convection complicates theoretical modeling of the diffusion. One must specify to what extent the flow is controlled by regular structures, such as granules, mesogranules, and supergranules, and in which ways it is random. Leighton (1964) introduced a very simple and instructive model that combines these elements. Here the surface diffusion is carried out by motions in a randomly renewing field of supergranules. The model assumes that the magnetic elements are carried passively to the boundary of a supergranule by its radial flow and remain there until the global supergranular flow field is renewed. As a result of this loss of flow memory the magnetic field elements perform normal "Brownian" random walks. The diffusion coefficient (or diffusivity) for normal diffusion is defined as one-fourth the mean squared displacement of flow markers per unit time: $K \equiv\left\langle r^{2}\right\rangle / 4 t$. The diffusion coefficient in Leighton's model is determined by the size and lifetime of the supergranules.

Leighton's idea has been tested observationally by measuring the motions of small magnetic fragments in time sequences of magnetograms (Mosher 1977; Schrijver \& Martin 1990) and elaborated theoretically and numerically (see Simon, Title, \& Weiss 1995; Ruzmaikin \& Molchanov 1997). Depending on how the aspect of randomness is incorporated, these elaborated models can show a richer variety of behavior than does the original Leighton version.

${ }^{1}$ acadavid@galileo.csun.edu.
In almost all previous work, the diffusion coefficient is assumed to be constant in time. However, when $K$ is calculated or estimated from analyses of solar data, values range between 70 and $1000 \mathrm{~km}^{2} \mathrm{~s}^{-1}$, depending on the type of observations or the model assumptions. Sheeley, Nash, \& Wang (1992) have found that a diffusivity of $600 \mathrm{~km}^{2} \mathrm{~s}^{-1}$ fits the observed latitudinal distribution of magnetic flux, the rigid rotation of coronal holes, and the $11 \mathrm{yr}$ polar reversals. In support of this value Simon et al. (1995) developed a series of kinematic models of diffusion generated by supergranulation and thereby constrained the value of the diffusion coefficient between $500 \mathrm{~km}^{2} \mathrm{~s}^{-1}$ and $700 \mathrm{~km}^{2} \mathrm{~s}^{-1}$. However, Schrijver \& Martin (1989), by directly tracking magnetic elements in plages and network, found diffusion coefficients of $120 \mathrm{~km}^{2} \mathrm{~s}^{-1}$ and $280 \mathrm{~km}^{2} \mathrm{~s}^{-1}$, respectively. From recent studies of $\mathrm{SOHO}$ MDI magnetograms, Hagenaar et al. (1999) concluded that the diffusion coefficient is smaller and that it varies with the scale: $70-90 \mathrm{~km}^{2} \mathrm{~s}^{-1}$ on short timescales $(\sim 1 \mathrm{hr})$ and $200-250 \mathrm{~km}^{2} \mathrm{~s}^{-1}$ on longer timescales $(\sim 5 \mathrm{hr})$. Berger et al. (1998b) find a diffusion coefficient $\sim 70 \mathrm{~km}^{2} \mathrm{~s}^{-1}$ for the transport of magnetic bright points. There is obviously a mismatch between the "directly observed" diffusivity and the diffusivity found by modeling.

One source of this difficulty may be that in most previous studies the more basic character of the diffusion, namely, the way the mean squared displacement grows with time $\left(\left\langle r^{2}\right\rangle \propto t^{\gamma}\right)$, was not taken into account: is it normal diffusion (i.e., $\gamma=1$ ), as usually assumed, or is it "subdiffusion" $(\gamma<1)$ or "superdiffusion" $(\gamma>1)$ ? Equivalently, if we define $\gamma \equiv 1$, is the diffusivity constant, or a decreasing or growing function of time?

While in the general physics literature there are plenty of examples of "anomalous" diffusion in different systems (Bouchaud \& Georges 1990; Weeks et al. 1994), there have been only a few attempts to identify the character of the solar diffusion (Lawrence 1991; Lawrence \& Schrijver 1993; 
Ruzmaikin et al. 1996 ). In the work of Lawrence \& Schrijver (1993) a diffusion exponent found to be $\gamma=0.89 \pm 0.20$ indicated possible subdiffusion for magnetic elements in active network tracked by Schrijver \& Martin (1990) for periods between $1 / 2$ and 5 days.

In addition, Lawrence \& Schrijver (1993) determined a second parameter governing the diffusion, namely, the effective dimension $D$ of the spatial distribution of the random walk sites. Mathematically, this dimension is not connected in a one-to-one relationship to $\gamma$ (O'Shaughnessy \& Procaccia 1985). $D$ is almost universally assumed to be the Euclidean dimension $(D=2)$; however, it was found for the Schrijver \& Martin (1990) data to be fractal: $D=1.56 \pm 0.08$, significantly excluding the Euclidean case. It was suggested (Schrijver et al. 1992; Balke et al. 1993; Lawrence \& Schrijver 1993) that the experimental results could be explained in terms of percolation theory since the number 1.56 characterizes the fractal dimension of a percolation pattern below threshold. A particle performing a random walk on that pattern would find its motion impeded by the finite clusters, a situation that would lead to subdiffusive behavior. In this paper we instead focus on details of the time evolution of the random walk.

In this work we analyze the $G$-band magnetic bright point (MBP) data of Berger \& Title (1996) and Berger et al. (1998a). As these authors explain, the relation between the $G$-band bright points and the underlying magnetic elements is subtle, since they have observed instances in which the $G$-band bright points are not observed or disappear when the associated magnetic elements are still observed. While this situation renders the bright-point data useless for the study of magnetic element lifetimes, however, it is very useful for the analysis of the random walk in which the whole process from splitting to merging can be followed. Another consideration is the fact that the magnetic field in the solar photosphere is a vector, and its motion may be affected by curvature forces as well as the magnetic force may well affect the convective motions. Thus, formally, we have no right to assume passive scalar diffusion. However this is a good first approximation to the diffusion of the line-of-sight component of the magnetic field (Parker 1979). In previous work (Cadavid et al. 1998) we found that the stochastic component of the velocity is closer to Kolomogorov-type turbulence, rather than Kraichnan. This may indicate that the effects of the magnetic field on the flow are small, which is consistent with the assumption of treating the magnetic field as a passive contaminant.

In order to correctly determine the diffusion parameters $\gamma$ and $D$ in a real physical system by following the random walk of passive markers (here magnetic elements), one needs observations of a large number of elements, each followed over many walk steps. The $G$-band magnetic bright-point data consist of approximately 1800 random walkers associated with magnetic elements, moving on granular scales, and with lifetimes of up to 70 minutes. These provide an ideal opportunity to perform this study.

In analyzing these data we find that the random walk steps do not occur at uniform intervals. Instead there are traps, i.e., sites in the photospheric flows (stagnation points), at which magnetic elements stick before the next displacement. The sticking times have a broad distribution function that extends to long periods. Further, we find that the random walks of the bright points, on timescales $\leq 20$ minutes, are significantly subdiffusive. Finally, we calculate the fractal dimension of the pattern of sites available to the random walkers. At short times, $D \rightarrow 1$, which is a reflection of the restricted motion in the intergranular lanes. For long times, $D \rightarrow 2$, which shows that as the granular pattern changes the restricted short time behavior is unimportant, and the walkers have access to the full underlying Euclidean space.

These three results can be unified in a natural way within a single analytical framework: the continuous time random walk (CTRW) model (Montroll \& Shlesinger 1984; Klafter, Blumen, \& Shlesinger 1987; Shlesinger \& Klafter 1989; Zumofen \& Klafter 1993). This approach takes into consideration both the effect of waiting times at stagnation points and the contribution from the transport due to the underlying convection. The CTRW method has been successfully used in the past to describe the dynamics of passive carriers diffusing in disordered media such as doped crystals and glasses (Alexander \& Orbach 1982; Blumen et al. 1986), and it is natural to apply it to the diffusion problem on the solar photosphere. We begin with the observation that the waiting times between random walk jumps of the MBPs follow what amounts to a truncated power law or one-sided Lévy distribution. The CTRW formalism then analytically leads, for timescales shorter than the truncation, to a subdiffusive spreading of walkers and to an effective fractal dimension $D<2$.

In solar physics the presence of power-law waiting times, or "fractal time" (Mandelbrot 1997), is implicit in the analysis of the magnetic observations by Smithson (1973), and in particular, in the modeling of Simon \& Weiss (1989) and Simon et al. (1995), who observed that after moving to the supergranular cell boundaries, the magnetic fields continue along the cell boundaries and end at accumulation "sinks." During the short, initial stage the transport is "ballistic," a trivial form of superdiffusion with $\left\langle r^{2}\right\rangle \propto t^{2}$ or $\gamma=2$. Subsequent displacement occurs only when the underlying cell pattern is renewed. In the version we will apply here to granular motions, the cells have radii $R$. Their radial outflows are randomly centered within distances given by a "tether" length: $0 \leq r \leq R_{\text {teth }}$ of points on a square grid. Each cell dies and is renewed individually. In the case that $R_{\text {teth }}>R$ we find that the flow pattern is completely decorrelated in a few cell lifetimes and that the diffusion is normal with $\gamma=1$ for long times. If $R_{\text {teth }}<R / 2$, the flow never decorrelates, there is no diffusion, and $\gamma=0$ for long times. However, there is an intermediate range of this control parameter, $R / 2<R_{\mathrm{teth}}<R$, where the behavior is critical: the flow pattern retains some memory of its configuration for many cell lifetimes, the sink sticking times take on a broad distribution, and the transport is subdiffusive with $0<\gamma<1$ for long times. It is this situation that we see for the granular transport of the magnetic bright points.

In $\S 2$ we perform a statistical analysis of the MBP data; this is followed, in $\S 3$, by a brief explanation of how the results are unified in the framework of the CTRW method. In $\S 4$ we run a version of the numerical model for renewing cells introduced by Simon et al. (1995). We end in $\S 5$ with a discussion of the various results.

\section{DIFFUSION OF SOLAR MAGNETIC BRIGHT POINTS}

The data set consists of a 70 minute sequence of $G$-band filtergrams obtained with the $50 \mathrm{~cm}$ Swedish Vacuum Solar Telescope on 1995 October 5. The pixel size is 0"083 (60.17 
$\mathrm{km}$ ), and the resolution is $\sim 0$ ". 4 . The observations correspond to a $29^{\prime \prime} \times 29^{\prime \prime}$ region of enhanced network near disk center. There are 1800 different MBPs, which are associated with magnetic elements. The duration of each series of single point displacements is determined both by the lifetime of the MBP and the time of observation. The images are corrected for seeing effects, and a subsonic filter is used to remove the acoustic and $f$-mode oscillations. The detailed description of the data can be found in Berger et al. (1998a).

In order to calculate the mean squared displacement we used all possible combinations in a given series. Thus a string of $n$ consecutive measurements of $\boldsymbol{r}$ for an MBP gives $(n-1)$ single interval displacement observations, $(n-2)$ double interval observations, ..., two $(n-2)$ interval observations, and one $(n-1)$ interval observation. Since these intervals overlap and thus are not independent, we have weighted each sample accordingly. The data were then averaged in logarithmic bins of width $\Delta \log t=0.06 \log$ (s) and a weighted linear least-squares fit of $\log \left\langle r^{2}\right\rangle$ versus $\log t$ was performed taking into account the uncertainties due to scatter in the bins. Figure 1 shows the results for the mean square displacement in the radial direction versus time. The dashed lines correspond to the linear fit over different ranges of time. The slopes of the lines and corresponding fitting range are $\gamma=0.76 \pm 0.04,0.3-22$ minutes; $\gamma=1.10 \pm 0.24,25-57.5$ minutes. We find subdiffusive transport for times shorter than 22 minutes and normal diffusion for times greater than 25 minutes. This result was presented in a different way by Berger et al. (1998b) in a plot of $\left(\left\langle r^{2}\right\rangle / t\right)$ versus $t$. They found a time-dependent diffusion coefficient that decreases up to $t \sim 26$ minutes, followed by a region of constant diffusion coefficient with value $50 \mathrm{~km}^{2}$ $\mathrm{s}^{-1}$ in the range 26-40 minutes, and finally a region of constant diffusion coefficient with value $79 \mathrm{~km}^{2} \mathrm{~s}^{-1}$ in the range 42-57 minutes. The regions of constant diffusion coefficient correspond to the regime of normal diffusion with $\gamma \sim 1$. Only in these regions can the ratio $\left\langle r^{2}\right\rangle / t$ yield a constant value. For completeness we have analyzed the mean squared displacement versus time in the $x$ and $y$ direc-

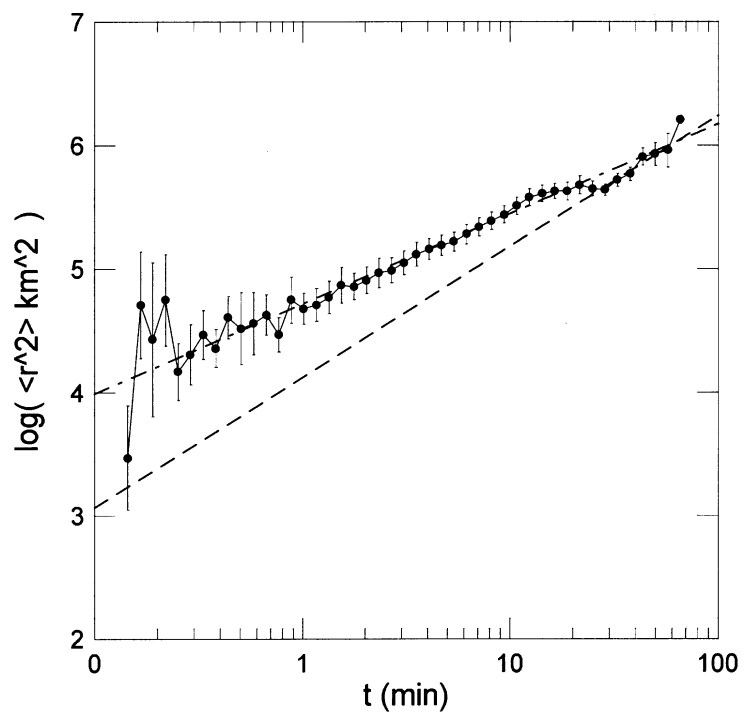

FIG. 1.- Mean squared displacement vs. time for data in logarithmic bins. The dashed lines present the linear fits in the ranges $0.3-22$ and 25-57.5 minutes. tions. We find the following values for the diffusion exponent: $x$-direction, $\gamma=0.69 \pm 0.03,0.3-57.5$ minutes; $y$-direction, $\gamma=0.83 \pm 0.05,0.3-22$ minutes; $y$-direction, $\gamma=1.10 \pm 0.30,22-57.5$ minutes. The scaling range for the $x$-direction is larger than for the $y$-direction. At $t \sim 22$ minutes the variance in the $y$-direction, which had been growing faster than in the $x$-direction, suddenly decreases. It is not clear what causes walkers to be constrained further in their motion in $y$ at this time. The measured asymmetry in the two variances may be due to some fundamental bias of the network, since the observed bright points are constrained to move in the intergranular lanes. Since the observations correspond to a small $\left(29^{\prime \prime} \times 29^{\prime \prime}\right)$ region, it is not surprising to encounter local anisotropies. The mean square displacement in the radial direction provides the best estimate, as it averages up these purely local effects.

After encountering a diffusion exponent that indicates subdiffusive transport, we proceeded to calculate the probability density of waiting times $\psi(t)$. The coordinates of the MBPs are defined by the centroid positions that change owing to evolution of the shape of the object or from motion. Because of this ambiguity, we have opted for a conservative definition in which a walker is determined to be waiting if the distance between two consecutive positions does not exceed 2 pixels. Furthermore, in order to define a waiting time we must be able to follow the MBP through a full jump-wait-jump cycle. The data presented in Figure 2 have been organized in bins of width 1 minute, and the error bars go as $1 / \sqrt{N}$, where $N$ is the number of points in the bin. For motion in the radial direction we find a scaling regime that terminates abruptly at $t \sim 14$ minutes.

From the last result, $\psi(t)$ has precisely the form of a truncated stable or Lévy distribution. Mathematically it can be described by

$$
\psi(t) \sim \begin{cases}t^{-\left(\gamma^{\prime}+1\right),} & t \leq \tau \\ 0, & t>\tau,\end{cases}
$$

where $\tau$ is the truncation time, which in Figure 2 occurs at $\sim 20$ minutes. We must point out that the truncation time

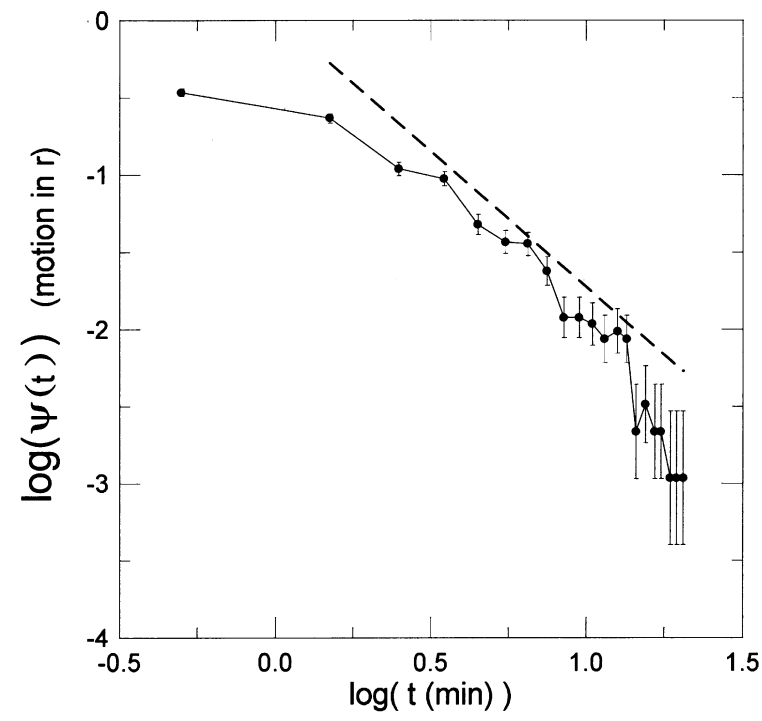

FIG. 2.- Log-log plot of the probability density of waiting times vs. time. The dashed line has a slope of $-(1+\gamma)$ for the value $\gamma=0.76$ obtained in Fig. 1. 


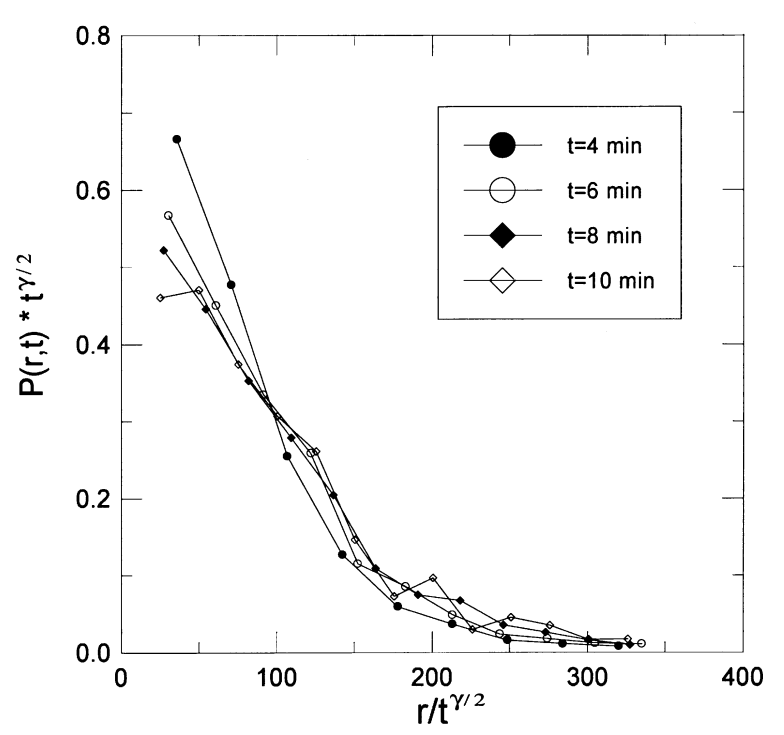

FIG. 3.-Probability density per unit area for being at position $r$ at a time $t$, vs. $r / t^{\gamma / 2}$. The different curves correspond to times $t=4,6,8$, and 10 minutes.

for $\psi(t)$ is comparable to the upper limit of the scaling range for subdiffusive transport in Figure 1. We will show in the next section that the parameter $\gamma^{\prime}$ is related to the diffusion exponent $\gamma$. For comparison with the value of the diffusion exponent previously obtained, we have included in Figure 2 a dashed straight line of slope -1.76 .

We then calculated $P(r, t)$, the probability density per unit area for being at a position $r$ at a time $t$. Figure 3 presents a graph of $P(r, t) t^{\gamma / 2}$ versus $\zeta=r / t^{\gamma / 2}$ for different times. The factor $t^{\gamma / 2}$ is included in order to be able to compare these results with the mathematical description in the next section. The temporal bins are centered at the given time $t$ and have a width of $40 \mathrm{~s}$ in each direction. While $P(r$, $t$ ) presents a cusp at small $r$ for short times $(4,6$, and 8 minutes), at approximately 10 minutes (the lifetime of the intergranular lane pattern) the cusp disappears. This implies that for times less than 10 minutes there is a large concentration of walkers close to the starting point. For larger times the walkers gradually cover uniformly the available underlying space. We must also note that the plots of $P(r, t)$ $t^{\gamma / 2}$ versus $\zeta$, for short times, converge to one curve, as will be shown in the mathematical model. This analysis can be complemented further by calculating the fraction $f(r, t)$ of walkers for which the displacement at time $t$ is less than $r$

TABLE 1

Dimension OF THE PATTERN OF Sites Available to the Random Walk FOR DIFFERENT TIMES

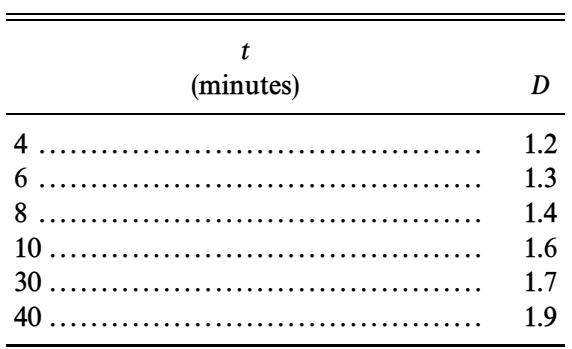

(Lawrence \& Schrijver 1993). For small $r / t^{\gamma / 2}$ there is a scaling regime in which

$$
\ln f \sim D \ln r+\text { constant, }
$$

where $D$ is the fractal dimension of the pattern of sites available to the random walk. By making linear fits to plots of $\ln f$ versus $\ln r$, we have found the following dimensions at the corresponding times (see Table 1).

While for short times the walkers, constrained to move in the intergranular lanes, have access only to a low dimensional pattern of sites, for longer times as the intergranular pattern renews itself, the walkers have access to essentially the two-dimensional underlying Euclidean space.

\section{THEORETICAL BACKGROUND}

As explained in $\S 1$, the observed phenomena can be naturally explained in the framework of the continuous time random walk (CTRW) method. This is not a thorough presentation of such a method, and we simply deal with those quantities that are relevant to the data. For further details the reader is directed to the extensive literature on this topic (Montroll \& Shlesinger 1984; Klafter et al. 1987; Shlesinger \& Klafter 1989; Zumofen \& Klafter 1993).

The motion of the MBPs is constrained by the intercellular lanes and the stagnation points. Fast motion occurs when the underlying convective cell pattern renews. The salient properties of this process can be described by a "jump model," in which random walkers wait at a trapping site before moving "instantaneously" to another location. To describe this process mathematically one needs to consider $\psi(t)$, the probability density to wait at a site a time $t$, and $p(r)$, the probability density for the jump, when it occurs, to have a length $r$. With these two functions one can calculate $P(r, t)$, the probability of being at $r$ at a time $t$. In turn, the mean square displacement can be obtained as

$$
\left\langle r^{2}(t)\right\rangle=\int d r r^{2} P(r, t) .
$$

Depending on the functional form of $\psi(t)$ the jump model can lead to normal or subdiffusive transport.

As we found in the data analysis section, the case of interest corresponds to a probability density $\psi(t)$ that asymptotically takes the form of a Lévy distribution (eq. [1]. Stable or Lévy distributions have proved to be useful in the description of anomalous diffusion (Klafter, Zumofen, \& Shlesinger 1994). Random variables are said to obey a stable distribution if their sum has the same distribution as the individual independent variables. While the best known example is the Gaussian distribution, it differs from the other stable distributions in that it does not obey an asymptotic power law and has finite moments.

Although in the real data the probability density is truncated at time $\tau$, to a first-order approximation, we present the results without truncation to illustrate the main points of the CTRW method. The mathematical form of $\psi(t)$ implies, first, that there is a scaling regime and, second, that there are walkers that wait very long times at the trapping sites. The combined effect leads to the concept of a "fractal time."

In this case the probability density $P(r, t)$, of being at a site $r$ at a time $t$, takes the form

$$
P(r, t) \sim t^{-\gamma^{\prime} / 2} f(\zeta),
$$


where $\zeta=r t^{-\gamma^{\prime} / 2}$ and $f(\zeta)$ is given by

$$
f(\zeta) \sim e^{-a_{1} \zeta-a_{2} \zeta^{2}}, \text { for small } \zeta,
$$

where $a_{1}, a_{2}$, and $a_{3}$ are functions of $\gamma^{\prime}$.

The exponential "cusp" at $\zeta \sim 0$ in $f(\zeta)$ indicates an accumulation of random walkers around the origin. This leads to $D<2$, for the dimension of the pattern of sites available to the random walkers, which was precisely the behavior observed in the data for times less than 10 minutes. For longer times the shape of $P(r, t)$ changes and, correspondingly, the dimension $D$ increases.

Finally the mean squared displacement is

$$
\left\langle r^{2}(t)\right\rangle \sim \begin{cases}t^{\gamma^{\prime}}, & 0<\gamma^{\prime}<1, \\ t / \ln (t), & \gamma^{\prime}=1, \\ t, & 1<\gamma^{\prime}<2 .\end{cases}
$$

As we have shown, when $\psi(t)$ has a power-law tail and no truncation, the parameter $\gamma^{\prime}$ can be identified with the diffusion exponent $\gamma$. For the data, in the subdiffusive regime where the probability density for waiting times exhibited a scaling law, we found $\gamma=0.76 \pm 0.04$ and $\gamma^{\prime}=0.61 \pm 0.09$. While the two numbers are in the same ball park, they are not the same for two main reasons. First, in the analytical calculation the probability distribution is not truncated as in the data. Second, the calculation of $\psi(t)$ from the data has ambiguities, and the number of samples is small as compared to the calculation of the mean squared displacement. We consider the latter calculation the more reliable result. We must stress, however, that both numbers clearly establish subdiffusive transport for times less than 20 minutes.

\section{NUMERICAL SIMULATION OF DIFFUSION IN A RENEWING CELLULAR PATTERN}

The CTRW formalism detailed above has the ability to give analytical results from a clear assumption. Thus, Lévy distributed waiting times for random walkers (fractal time) leads to subdiffusion. The formalism, however, offers no physical basis for the assumption of fractal time. In this section we show that both this and the resulting subdiffusive transport can arise naturally in a simple, intuitive, and quite familiar model.

In order to illustrate the process described above, we have revisited a numerical model introduced by Simon et al. (1995) to describe the dynamics of diffusion at supergranular scales. At these scales observations show the migration of the magnetic flux from the point where it emerges within a supergranule to the cell boundary where it is constrained to move on the supergranular lanes and eventually accumulates at sinks of the flow field. As the supergranular pattern evolves, the sinks displace randomly. While the MBPs are only observed at the intergranular lanes, we find that once there the phenomena at granular and supergranular scales have many properties in common.

The version of the model of Simon et al. (1995) that we make use of here assumes a square grid of $(x, y)$ locations separated by distance $2 R$. Cells of radius $R$ are tethered to the grid centers at distances $0 \leq r \leq R_{\text {teth }}$ chosen at random from a uniform distribution and in azimuthal directions uniformly distributed from 0 to $2 \pi$. The cell lifetimes have average value $T$ and are uniformly distributed between $2 T / 3$ and $4 T / 3$. As each cell dies it is replaced by a new one with newly selected lifetime, tether length, and azimuth. At the center of each cell is a source of fluid that flows to the intercellular lanes, where it adds vectorially with the flow from other cells. We take the flow from each source to be radial, with form (Simon \& Weiss 1989)

$$
v(r) \sim(V r / R) e^{-(r / R)^{2}} .
$$

We take the peak velocity to be $V \sim R / T$.

Walkers are randomly placed on this pattern, and their motions under the influence of the net flow are recorded over many cell lifetimes. Figure 4 displays $\left\langle r^{2}\right\rangle$ versus $t$ for different values of $R_{\text {teth }}$ as compared to the cellular radius.

For times less than the average cell lifetime we find the exponent $\gamma \sim 2$. This is a trivial form of superdiffusion. It corresponds to "ballistic" motion and is due to the unidirectional advection of the passive markers toward the cell boundary. This process is seen in the results of Lawrence \& Schrijver (1993) for the random walks of magnetic elements in network on timescales $\sim 3-7 \mathrm{hr}$. It also has been seen at similar timescales by Hagenaar et al. (1999) when studying the random walk of magnetic flux concentrations from high-resolution magnetograms obtained by $\mathrm{SOHO}$. We do not see the effect, however, when studying diffusion at granular scales. Here magnetic elements do not become visible as bright points until they are situated in the intergranular lanes.

When the markers reach the cell edges, they are carried along the intercellular lanes until they reach flow sinks located at some of the cell vertices. Further motion relies on renewal of the flow pattern following the deaths of individual cells. The nature of this motion depends on the value of the tether length relative to the cell radius. Thus for time intervals longer than a cell lifetime we find the following: (1) for $R_{\text {teth }}<0.5 R$, no diffusion $(\gamma=0)$; (2) for $0.5 R<R_{\text {teth }}$ $<R$, subdiffusion $(0<\gamma<1)$; and (3) for $R_{\text {teth }}>R$, normal diffusion $(\gamma=1)$. These results are presented first in Figure 4 , in a $\left\langle r^{2}\right\rangle$ versus $t$ diagram and then in Figure 5, which presents the diffusion exponent $\gamma$ as a function of tether length.

The interpretation of this result is straightforward. If the tether length is short, new cells will be born at almost the same location as the old ones and the pattern of flow sinks

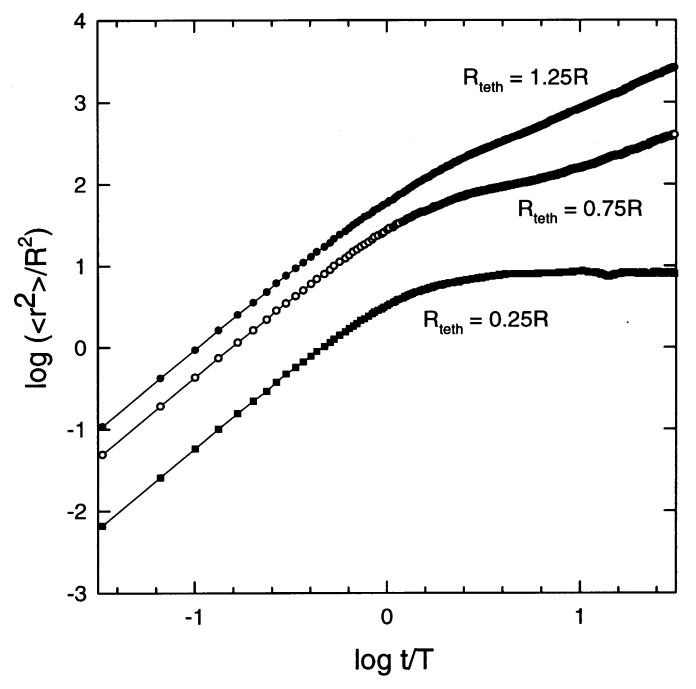

FIG. 4.-Mean squared displacement vs. time for the numerical model. The different curves correspond to tether lengths $R_{\text {teth }}=0.25 R, 0.75 R$, and $1.25 R$, where $R$ is the cellular radius. 


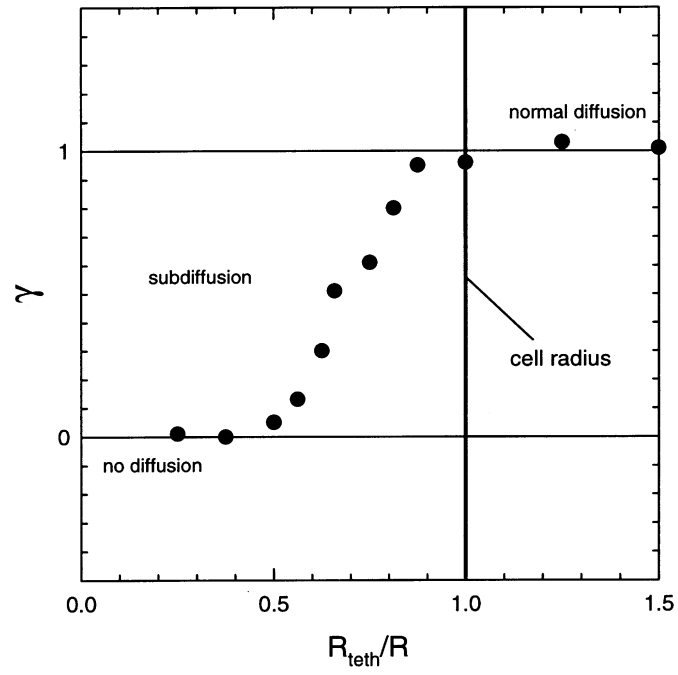

Fig. 5.-Variation of the diffusion exponent $\gamma$ as a function of the tether length in the numerical model.

never changes. Then the walkers are permanently trapped. If the tether length is long, then the changing cell structures easily disrupt the pattern of sinks and the walkers diffuse normally. Between these two cases is a zone $(0.5 R<$ $R_{\text {teth }}<R$ ) of critical behavior in which walkers are displaced but also can be trapped for periods on all timescales. This is the counterpart of the Lévy distribution of waiting times (fractal time) introduced in the previous section. Here, as there, it leads to subdiffusive behavior. We note that in these cases, when steps do occur in the $x$ - or $y$-directions, their lengths are clustered around integer multiples of $2 R$.

In this phenomenological model the control parameter is $R_{\text {teth }} / R$. The results can be interpreted physically in terms of the spatial correlation between old and new cell patterns or the "memory" of the underlying convection. At one extreme, when the new pattern is totally correlated to the old one, the random walkers remain in the traps and there is

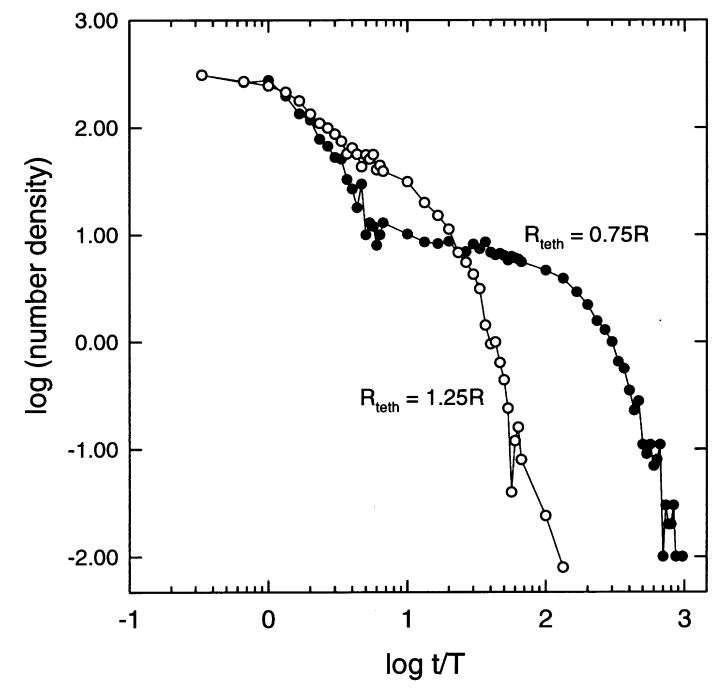

FIG. 6.- - Log-log plot of the number density of waiting times vs. time for the numerical model. The two curves correspond to tether lengths $R_{\text {teth }}=0.75 R, 1.25 R$, where $R$ is the cellular radius. no motion. At the other extreme, when there is no correlation between the old and new cellular patterns the motion is totally random and the diffusion is normal. In the intermediate range, which corresponds to the MBP data, the transport is subdiffusive.

Figure 6 presents the number density of waiting times versus time in cell lifetimes. The case $R_{\text {teth }}>R$, corresponding to normal diffusion, shows a scaling range followed by a fast decrease indicating a truncation in the number density of waiting times. For $R_{\text {teth }}<R$ the scaling range is less steep and the truncation occurs at much larger times.

Finally, in the critical range $\left(0.5 R<R_{\text {teth }}<R\right)$, examination of the displacement of random walkers from their starting points does indicate the effect of fractal dimension $D<2$ for the sites available for motion. In this case the effect can be attributed to the restriction of the motion to the square grid defined by the intercellular lanes.

\section{DISCUSSION}

We have found that the commonly assumed normal (Brownian) model for random walks of magnetic elements on the Sun does not always apply. We have demonstrated the effect on the Sun by tracing the observed motions of $G$-band bright points on granular spatial and temporal scales. For timescales less than 20 minutes we find three results: (1) subdiffusive transport; (2) fractal time, that is, a broad, roughly power-law distribution of walker waiting times; and (3) an effective fractal dimension $D<2$. By applying to the solar case the CTRW formalism developed for analytical treatment of anomalous transport in disordered media, we have shown the close logical connection between these three phenomena. And finally, by application of the "tether" model of random walks in a field of renewing convective cells we have found that the origin of the effect appears to be a long but finite retained memory in the granular pattern.

Magnetic bright points become observable only when magnetic flux is concentrated in the intergranular lanes (Muller \& Hulot 1989; Title et al. 1992). While in the case of the granulation, the bright-point data do not show the migration of the magnetic flux to the boundary, once the walkers are on the boundaries, there are many similarities between the transport and evolution at granular and supergranular scales. This includes the waiting times and the corresponding subdiffusive transport. When the underlying granulation pattern changes, the MBP's are given a push that can be identified as a jump in the context of the CTRW model. For longer times (several lifetimes of the granulation pattern) the waiting times become unimportant and the motion of the MBPs is dominated by the random evolution of the granulation pattern, leading to normal transport.

The renewing cell model we have used was originally introduced by Simon et al. (1995) to study supergranular diffusion, the velocity distribution in equation (7) was derived from observations of mesogranules (Simon \& Weiss 1989), and in $\S 4$ we applied the model to granules. This mixing of metaphors should not detract from the point of $\S$ 4 , that apparently abstract notions like fractal time and anomalous diffusion can arise from a simple, concrete model. The model also demonstrates a degree of robustness for the ideas. Analytical derivation of the subdiffusion in $\S 3$ requires a power-law form of the distribution of waiting times. Our model, as shown in Figure 6, reproduces this 
only qualitatively. Nevertheless, subdiffusion is found. The key feature is a long tail on the distribution extending to waiting times longer than the period of observations. In turn this can also be interpreted in terms of the underlying convection. The cellular pattern is neither fixed as in Rayleigh-Bénard cells, nor is it totally uncorrelated from the old pattern as required in Leighton's model. We find instead a behavior between these extremes.

We thank T. Berger for generously sharing the excellent bright-point data and for useful comments. This work was supported in part by NSF grant ATM-9628882.
Alexander, S., \& Orbach, R. 1982, J. de Phys., 43, L625

Balke, A. C., Schrijver, C. J., Zwaan, C., \& Tarbell, T. D. 1993, Sol. Phys., 143,215

Berger, T. E., Löfdahl, M. G., Shine, R. A., \& Title, A. M. 1998a, ApJ, 495, 973

. 1998b, ApJ, 506, 439

Berger, T. E., \& Title, A. M. 1996, ApJ, 463, 365

Blumen, A., Klafter, J., \& Zumofen, G. 1986, in Optical Spectroscopy of Glasses, ed. I. Zschokke (Dordrecht: Reidel), 199

Bouchaud, J. P., \& Georges, A. 1990, Phys. Rep., 195, 127

Cadavid, A. C., Lawrence, J. K., Ruzmaikin, A. A., Walton, S., \& Tarbell, T. 1998, ApJ., 509, 918

Hagenaar, H. J., Schrijver, C. J., Title, A. M., \& Shine, R. A. 1999, ApJ, 511, 932

Klafter, J., Blumen, A., \& Shlesinger, M. F. 1987, Phys. Rev. A, 35, 3081

Klafter, J., Zumofen, G., \& Shlesinger, M. F. 1994, in Lévy Flights and Related Topics in Physics, ed. M. F. Shlesinger, G. Zaslavsky, \& U. Frisch (Berlin: Springer), 196

Lawrence, J. K. 1991, Sol. Phys., 135, 249

Lawrence, J. K., \& Schrijver, C. J. 1993, ApJ, 411, 402

Leighton, R. B. 1964, ApJ, 140, 1574

Mandelbrot, B. B. 1997, Fractals and Scaling in Finance (New York: Springer)

Montroll, E. W., \& Shlesinger, M. F. 1984, in Nonequilibrium Phenomena II: From Stochastics to Hydrodynamics, ed. J. L. Lebowitz \& E. W. Montroll (Amsterdam: North-Holland), 1

\section{REFERENCES}

Mosher, J. M. 1977, Ph. D. thesis, California Inst. Tech.

Muller, R. T., \& Hulot, J. C. 1989, Sol. Phys., 119, 229

O’Shaughnessy, B., \& Procaccia, I. 1985, Phys. Rev. Lett., 54, 455

Parker, E. 1979, Cosmic Magnetic Fields (Oxford: Oxford Univ. Press)

Ruzmaikin, A. A., Cadavid, A. C., Chapman, G., Lawrence, J. K., \& Walton, S. 1996, ApJ, 471, 1022

Ruzmaikin, A. A., \& Molchanov, S. A. 1997, Sol. Phys., 173, 223

Schrijver, C. J., \& Martin, S. F. 1990, Sol. Phys., 129, 95

Schrijver, C., Zwaan, C., Balke, A., Tarbell, T., \& Lawrence, J. 1992, A\&A, 253, L1

Sheeley, N. R., Nash, A. G., \& Wang, Y. M. 1992, ApJ, 401, 378

Shlesinger, M. F. \& Klafter, J. 1989, J. Phys. Chem., 93, 7023

Simon, G. W., Title, A. M., \& Weiss, N. O. 1995, ApJ, 442, 886

Simon, G. W., \& Weiss, N. O. 1989, ApJ, 345, 1060

Smithson, R. C. 1973, Sol. Phys., 29, 365

Title, A. M., Topka, K. P., Tarbell, T. D., Schmidt, W., Balke, C., \& Scharmer, G. 1992, ApJ, 393, 782

Weeks, E., Solomon, T., Urbach, S., \& Swinney, H. 1994, in Lévy Flights and Related Topics in Physics, ed. M. Shlesinger, G. Zaslavsky, \& U. Frisch (Berlin: Springer), 51

Zumofen, G., \& Klafter, J. 1993, Phys. Rev. E, 47, 851 